\title{
ESTUDO DE UTILIZAÇÃO DE MEDICAMENTOS EM UMA UNIDADE HOSPITALAR DE CAARAPÓ-MS
}

\section{STUDY OF USE OF MEDICINES IN A HOSPITAL OF CAARAPÓ-MS}

\author{
Adriana Mary Mestriner Felipe de Melo'; Edilene Alves dos Santos², Marcos Ávalos \\ Berndt $^{3}$, Luis Arthur Castilho ${ }^{4}$
}

1 - Doutora em Ciências da Saúde (UnB), Mestre em Microbiologia (UEL), docente do Centro
Universitário da Grande Dourados (UNIGRAN)
2 - Farmacêutica, Especialista em Saúde Pública pela Escola de Saúde Pública David Nasser,
Prefeitura Municipal de Dourados
3 - Farmacêutico, Mestre em Ciências Farmacêuticas (UFMS), Prefetura Municipal de Nova Alvorada 4
4 - Farmacêutico, Mestre em Ciências da Saúde (UFGD)

\section{RESUMO:}

O Uso correto e Seguro de Medicamentos está sempre sendo uma das temáticas em discussão quando o assunto é evitar gastos e efeitos indesejados de medicamentos. Como forma de ampliar o conhecimento da realidade sobre o assunto, os estudos de utilização de medicamentos é uma das ferramentas que pode ser utilizada e explorada. Neste estudo objetivou-se avaliar o uso de medicamentos em um hospital público da cidade Caarapó, empregando os Indicadores Selecionados de Uso de Medicamentos estabelecidos pela OMS. Foram analisadas as fichas de Prescrição e Evolução médica de 133 pacientes internados no Hospital. Todos os pacientes pesquisados estiveram internados e receberam pelo menos 1 medicamento durante o período de hospitalização. Apesar da prescrição de medicamentos prescritos em uma unidade hospitalar da cidade de Caarapó seguir alguns dos critérios estabelecidos pela Organização Mundial de Saúde, ainda existem inúmeros indicadores que precisam ser melhorados.

Palavras-chave: Medicamentos; Medicamentos essenciais; Saúde pública.

\begin{abstract}
:
The correct and safe use of medicines is always one of the topics under discussion when it comes to avoiding unwanted expenses and effects of medicines. As a way of expanding the knowledge of the reality on the subject, studies on the use of medicines is one of the tools that can be used and explored. This study aimed to evaluate the use of medicines in a public hospital in the city of Caarapó, using the Selected Indicators of Use of Medicines transformed by WHO. Were analyzed as prescription and medical records of 133 patients admitted to the Hospital. All patients surveyed were hospitalized and receiving at least 1 medication during the hospitalization period. Despite the prescription of drugs prescribed in a hospital in the city of Caarapó following some of the criteria defined by the World Health Organization, there are still numerous indicators that need to be improved.
\end{abstract}

Keywords: Medicines; Essential drugs; Public health.

\section{INTRODUÇÃO}

A Organização Mundial de Saúde diz que há uso racional de medicamentos quando 
pacientes recebem medicamentos apropriados para suas condições clínicas, em doses adequadas às suas necessidades individuais, por um período adequado e ao menor custo para si e para a comunidade (ROCHA, 2014).

Os estudos envolvendo a utilização de medicamentos constitui uma das ferramentas para conhecer a realidade sobre o assunto considerando diferentes contextos (idade, local onde a medicação é adquirida, fatores de adesão ao tratamento, dentre outros) e, portanto, constitui um dos caminhos para a mudança tão almejada nas práticas profissionais envolvendo a cadeira de medicamentos (LEITE; VIEIRA; VEBER, 2008).

O Uso Racional de Medicamentos (URM), atualmente, surge como uma enérgica maneira de se promover, proteger e recuperar a saúde dos indivíduos. Está relacionada à correta prescrição, dispensação e utilização dos medicamentos, a preços acessíveis, observando sempre sua segurança, eficácia e qualidade (OSORIO-DE-CASTRO, 2014).

Diante deste quadro, a Organização Mundial de Saúde já estabeleceu como grande desafio para a próxima década uma melhoria na racionalidade do uso de medicamentos, havendo a necessidade de promoção da avaliação desse uso e a vigilância de seu consumo (AQUINO, 2008).

O sucesso terapêutico no tratamento de doenças depende de bases que permitam a escolha do tratamento, medicamentoso e/ou não medicamentoso, a seleção do medicamento de forma científica e racional, considerando sua efetividade, segurança e custo, bem como a prescrição apropriada, a disponibilidade oportuna, a dispensação em condições adequadas e a utilização pelo usuário de forma adequada. Dessa forma, as decisões clínicas e as relações estabelecidas entre os profissionais e usuários são determinantes para a efetividade terapêutica (CALADO, 2016.).

Mesmo com a recomendação dos padrões para uma prescrição racional, poucos dados estão disponíveis no Mato Grosso do Sul sobre as condições reais das prescrições médicas.

Como se pode notar, o uso racional de medicamentos envolve uma série de fatores complexos e interdependentes. No entanto, é de extrema importância que este seja alcançado, tendo em vista as graves conseqüências que o uso irracional de medicamentos pode trazer para toda a população. Considerando que o conhecimento das práticas sobre a prescrição de medicamentos pode auxiliar na elaboração de estratégias dentro das comissões presentes dentro do ambiente hospitalar, o objetivo deste estudo foi avaliar o uso de medicamentos em um hospital público da cidade Caarapó, empregando os Indicadores Selecionados de Uso de Medicamentos estabelecidos pela OMS. 


\section{MATERIAL E MÉTODOS}

Trata-se de um estudo do tipo descritivo-exploratório, com delineamento de pesquisa documental, que objetiva a identificação de uma temática e o seu conhecimento nos limites de uma realidade específica (GIL, 2002). Desse ponto de vista, os prontuários médicos que foram utilizados como objetos de análise, se enquadram nessa classificação. O protocolo foi submetido do CEP-UNIGRAN e aprovado para execução (236/07) de acordo com as normativas Nacionais envolvendo Pesquisas com Seres humanos estabelecidas pela CONEP.

Foram estudados, quanto à racionalidade terapêutica, os indicadores de prescrição dos prontuários dos pacientes internados em um hospital da cidade de Caarapó com base em indicadores de prescrição detalhados no formulário padrão utilizado e padronizado por Ferreira (2005). O Hospital é um hospital Geral com atenção ambulatorial em média complexidade e internação, atendendo urgência e emergência.

Foram estudados os prontuários médicos (que contêm dados das prescrições médicas) dos pacientes que foram atendidos num período de um ano, na forma retrospectiva, compreendendo os meses de fevereiro a julho/2008, quanto à racionalidade terapêutica, com base em indicadores de prescrição detalhados no formulário padrão utilizado e padronizado por Ferreira (2005). A escolha da amostra da pesquisa foi realizada sorteando-se uma semana de cada um dos meses que compreendem a pesquisa, e para cada semana, foram sorteados três dias e um turno específico. Em cada um dos dias escolhidos para a pesquisa, 7 ou 8 prontuários de pacientes internados foram estudados, garantindo uma média de 22,16 pacientes/mês, com um total de 133 prontuários, perfazendo uma média de 300 participantes. Foram excluídos pacientes com idade inferior a 18 anos, com alterações neurológicas de caráter psicomotor ou dificuldade de compreensão de comandos verbais. Não foram incluídos os pacientes que se recusarem a participar da pesquisa ou aqueles que foram atendidos por médicos que não aceitaram ter sua prescrição analisada.

O instrumento de coleta dos dados do estudo foi constituído por formulário preenchido pelo pesquisador, a partir das prescrições dos pacientes, obtidas por meio do prontuário hospitalar. Optou-se por avaliar o impacto do uso racional de medicamentos por meio dos indicadores de prescrição ao nível hospitalar (WHO, 2002; 2005, 2006), relacionados a seguir: 
1- Número de medidas não-medicamentosas por prescrição, listadas em prontuário ou receita médica.

2 - Número médio de medicamentos por prescrição, relacionados em prontuário ou receita médica.

3 - Número de medicamentos prescritos por paciente, introduzidos no mercado há menos de 5 anos.

4 - Número de medicamentos prescritos com o nome genérico por paciente.

5 - Número de antibióticos por prescrição.

6 - Número de prescrições com indicação de, pelo menos, um medicamento injetável.

7 - Número de medicamentos prescritos em esquema de demanda (se necessário), por 8 prescrição hospitalar.

8 - Número de medicamentos por prescrição que se encontram na Relação Nacional de Medicamentos do Brasil (BRASIL, 2002).

9 - Número de medicamentos prescritos, por paciente, que se encontram na $15^{\mathrm{a}}$ Lista Modelo de Medicamentos Essenciais da Organização Mundial da Saúde.

10 - Número de medicamentos prescritos disponibilizados pelo sistema público de saúde.

11 - Presença dos seguintes itens na prescrição: nome e endereço do prescritor, data, concentração/dosagem, forma de administração, quantidade total a ser dispensada, forma farmacêutica, instruções, advertências, assinatura do prescritor, nome e endereço do paciente.

Os dados foram reunidos e codificados em banco de dados por meio do Programa Epi Data, versão 1.5 (LAURITSEN, 2001), bem como a análise estatística desses dados, com o auxílio do Software Epi-info, versão 6.0 (DEAN et al., 1994). Os dados foram expressos como freqüência absoluta ou relativa, média + desvio padrão (para variáveis paramétricas) ou mediana e percentis 25 e 75 (para variáveis não-paramétricas).

Os dados foram coletados diretamente dos prontuários hospitalares referentes aos pacientes que atenderam a todos os critérios de inclusão e exclusão. Estes pacientes utilizaram-se dos serviços prestados pelo hospital durante o período que compreende os meses de fevereiro a julho (período escolhido para pesquisa).

Para a coleta de dados foi sorteada uma semana de cada um dos meses que compreendem a pesquisa. Para cada semana, foram sorteados três dias e um turno específico dos quais os dados serão coletados, conforme detalhado anteriormente. 
Após esta etapa, para os pacientes sorteados que aceitaram participar da pesquisa e assinaram o TCLE, a ficha de coleta de dados foi preenchida, pela pesquisadora (considerando-se todos os medicamentos prescritos naquele dia). No caso de alterações da prescrição para o mesmo medicamento, ao longo do dia, considerou-se a última modificação.

\section{RESULTADOS E DISCUSSÃO}

Foram analisadas as fichas de Prescrição e Evolução médica de 133 pacientes internados de fevereiro a julho na cidade de Caarapó - MS. Todos os pacientes pesquisados estiveram internados e receberam pelo menos 1 medicamento durante 0 período de hospitalização.

A idade dos pacientes variou de 18 a 102 anos, com média de 56,6 anos. Quase $50 \%$ dos pacientes constituem população idosa com 60 anos ou mais. A maioria pertencia ao sexo feminino $(57,9 \%)$ e apresentava um diagnóstico de doença $(98,5 \%)$.

Dentre as patologias dos pacientes pesquisados foram classificadas de acordo com a nomenclatura CID (Código Internacional de Doenças), que é um sistema de categorias atribuídas a entidades mórbidas ou um conjunto de doenças semelhantes. É atribuída categoria única a cada estado de saúde, à qual corresponde um código, que pode ter até 6 caracteres sendo as mais frequentes as doenças do aparelho respiratório e circulatório, Doenças endócrinas, nutricionais e metabólicas, doenças do aparelho digestivo, Doenças do sistema nervoso e infecções de uma maneira geral.

Os medicamentos prescritos foram classificados de acordo com o primeiro nível de classificação da ATC, tendo como maiores porcentagens: medicamentos pertencentes ao grupo de "Sistema digestivo e metabolismo" (A) (37,7\%), ao grupo de "Sistema Cardiovascular" (C) (15,2\%), ao grupo de "Anti-infecciosos de uso sistêmico" (J) (9,2\%), ao grupo de "Sistema Nervoso" (N) (18,2\%) e ao grupo de "Sistema Respiratório" (R) (11\%).

Os grupos restantes corresponderam a $8,7 \%$ dos casos, incluindo medicamentos para o "Sistema geniturinário e hormônios sexuais", "Sangue e órgãos hematopoiéticos", "Medicamentos dermatológicos", "Hormônios de uso sistêmico, exceto os sexuais", "Sistema musculoesquelético", "Ilegíveis" e "Não classificados pelo sistema ATC".

Dos 695 medicamentos prescritos: 42,4\% destes continham a forma farmacêutica, $82,7 \%$ apresentavam dose e $84,8 \%$ apresentavam posologia, 84,8\% continham o intervalo de administração a ser adotado, 77,7\% apresentavam a via de administração a ser usada, 
das quais $20,9 \%$ tratava-se de via oral, $11,6 \%$ via intravenosa e $0,6 \%$ via intramuscular, sendo $58,4 \%$ destas classificadas como injetáveis. Outras vias que não as citadas corresponderam a 5\% (TABELA 1).

Tabela 1 Análise dos parâmetros de prescrição dos 534 medicamentos analisados.

\begin{tabular}{cccc}
\hline Parâmetros analisados & Informação & $\mathbf{N}$ & $\%$ \\
\hline \multirow{2}{*}{ Forma farmacêutica } & Presente & 295 & 42,4 \\
Posologia & Prescrição de injetáveis & 406 & 58,4 \\
& Presente & 209 & 30,1 \\
& Presença de dose & 575 & 82,7 \\
Via de administração & Presença de intervalo & 474 & 84,8 \\
& Presente & 540 & 77,7 \\
& Prescrição de via intravenosa & 63 & 11,6 \\
& Prescrição de via endovenosa & 335 & 61,9 \\
\hline
\end{tabular}

\section{$\mathrm{N}$ : número de medicamentos que continham a informação requerida.}

Considerando que a prescrição acontece de forma manual e que muitos possuem caligrafia de difícil compreensão, destaca-se esse como um fator que pode interferir no uso correto e seguro de medicamentos. Alguns estudos defendem a substituição da prescrição manual pela eletrônica, onde o prescritor ao invés de escrever a prescrição manualmente, utilizaria um computador para digitá-la, de acordo com um modelo já estabelecido, evitando desta forma os erros provenientes do não entendimento gráfico da prescrição, além de evitar rasuras, que dificultam ainda mais a leitura, pois os erros podem ser corrigidos pelo prescritor antes da impressão (MIASSO et al., 2009; GIMENES et al., 2011.).

A posologia é uma informação imprescindível em qualquer prescrição, sendo que a mesma deveria sempre ser feita de maneira completa, ou seja, contendo a concentração, a via de administração e o intervalo de uso, pois dela depende a ação farmacológica. No presente estudo, a presença de posologia foi completa em um percentual de $30,1 \%$, inferior 
aos valores encontrados no Rio de Janeiro, de 59,91\% (PECLY et al., 2006).

Dos medicamentos prescritos, $86,3 \%$ o foram pela denominação genérica, $75,1 \%$ pertenciam à REMUME, 81,7\% à RENAME e 59,7\% à Lista Modelo da OMS. Observou-se ainda que $91,1 \%$ dos medicamentos prescritos eram considerados como agentesmedicamento padronizado dentro do hospital. A TABELA 2 apresenta os valores encontrados na amostra estudada no que se refere aos indicadores de prescrição propostos pela OMS, de modo a atender ao objetivo formulado pela presente pesquisa.

A porcentagem de prescrições com presença de antibióticos foi de 44,36\% (tabela 2).

Tabela 2 - Medicamentos prescritos em uma unidade hospitalar da cidade de Caarapó (MS).

\section{Grupos ou agentes farmacológicos}

\section{Número de receitas em que foram prescritos}

$(\%)$

\begin{tabular}{lll}
\hline Paracetamol (N02BE01) & 14 & 2,0 \\
Diazepam (N05BA01) & 15 & 2,2 \\
Aminofilina (R03DA05) & 16 & 2,3 \\
Fenoterol (R03AC04) & 17 & 2,4 \\
Ampicilina (J01CA01) & 17 & 2,4 \\
Furosemida (C03CA01) & 17 & 2,4 \\
Dexametasona (C05AA09) & 17 & 2,4 \\
Ácido ascórbico (A11GA) & 25 & 3,6 \\
Captopril (C09AA01) & 25 & 3,6 \\
Cefalotina (J01DB03) & 28 & 4,0 \\
Ambroxol (R05CB06) & 28 & 4,0 \\
Escopolamina (A04AD01) & 33 & 4,7 \\
Metoclopramida (A03FA01) & 44 & 6,3 \\
Ranitidina (A02BA02) & 49 & 7,1 \\
Metamizol (N02BB02) & 58 & 8,3 \\
Complexo B (A11EA) & 65 & 9,4 \\
\hline
\end{tabular}

A média de medicamentos prescritos por prescrição foi de cinco a seis $(38,28,6 \%)$, entre três e quatro $(37,27,8)$ ou de sete a oito $(25,18,8 \%)$. De acordo com a OMS a 
associação desse número de medicamentos por prescrição precisa ser revista. Já em relação aos dados do paciente, prescritor, assinatura foram dados encontrados em todos os prontuários.

\section{CONCLUSÃO}

Apesar da prescrição de medicamentos prescritos em uma unidade hospitalar da cidade de Caarapó seguir alguns critérios estabelecidos pela Organização Mundial de Saúde, ainda existem inúmeros indicadores que precisam ser melhorados.

\section{REFERÊNCIAS}

AQUINO, Daniela Silva de. Por que o uso racional de medicamentos deve ser uma prioridade?. Ciência \& Saúde Coletiva, v. 13, p. 733-736, 2008.

BRASIL. Ministério da Saúde. Secretaria de Políticas de Saúde. Departamento de Atenção Básica. Gerência Técnica de Assistência Farmacêutica. Relação Nacional de Medicamentos Essenciais - RENAME. 3 ed. Brasília: Ministério da Saúde, 2002.

CALADO, Deuzimar Cordeiro. Orientação ao usuário quanto ao uso racional de medicamentos e sua inutilização quando necessário. 2016.

CASSIANI, S. H. B.; FREIRE, CLÁUDIA C. F.; GIMENES, R. E. A prescrição médica eletrônica em um hospital universitário: falhas de redação e opiniões de usuários. Rev. Esc. Enferm. USP, São Paulo, v. 37, n. 4, p. 51-60, 2003.

CONSELHO REGIONAL DE FARMÁCIA DO ESTADO DE SÃO PAULO. Farmácia Hospitalar. Abril/2007. Disponível em <http:www.crfsp.org.br>. Acessado em: 5 de abril de 2008.

DEAN, A.G.; DEAN, J.A.; COULOMBIER, D.; BRENDEL, K.A.; SMITH, D.C.; BURTON, A.H.; DICKER, R.C.; SULLIVAN, K.; FAGAN, R.F.; ARNER, T.G. Epi Info, Version 6: A Word Processing Database and Statistics Program for Epidemiology on Microcomputers. Atlanta, Centers of Disease Control and Prevention, 1994. 
FERREIRA, M.B.C. (Coord.). Estudo Multicêntrico para Avaliação de Indicadores do Uso Racional de Medicamentos. Porto alegre: Universidade Federal do Rio Grande do Sul, 2005.

GIL, A.C. Como elaborar projetos de pesquisa. 4.ed. São Paulo: Atlas, 2002.

GIMENES, Fernanda Raphael Escobar et al. Medication wrong-route administrations in relation to medical prescriptions. Revista latino-americana de enfermagem, v. 19, n. 1, p. 11-17, 2011.

LAURITSEN, J.M.; BRUUS, M.; MYATT, M.A. EpiData, Version 2.1a. An Extended Tool for Validated Entry and Documentation of Data. Odense, Denmark: The EpiData Association, 2001.

LEITE, Silvana Nair; VIEIRA, Mônica; VEBER, Ana Paula. Estudos de utilização de medicamentos: uma síntese de artigos publicados no Brasil e América Latina. Ciênc. saúde coletiva, Rio de Janeiro , v. 13, supl. p. 793-802, abr. 2008.

MIASSO, Adriana Inocenti et al. Prescription errors in Brazilian hospitals: a multi-centre exploratory survey. Cadernos de saúde pública, v. 25, n. 2, p. 313-320, 2009.

OMS. INTERNATIONAL SOCIETY OF DRUG BULLETINS AND WORLD HEALTH ORGANIZATION. Starting or Strengthening a Drug Bulletin - A Practical Manual, 2005.

ORGANIZAÇÃO MUNDIAL DE SAÚDE. Promovendo o Uso Racional de Medicamentos: Componentes Centrais. Geneva: OMS, 2002.

OSORIO-DE-CASTRO, Claudia Garcia Serpa et al. (Ed.). Assistência farmacêutica: gestão e prática para profissionais da saúde. SciELO-Editora FIOCRUZ, 2014.

PECLY, J. S. et. al.. Análise qualitativa de receitas ambulatoriais em dado período em um hospital da rede pública: possíveis fatores que podem influenciar o uso correto de medicamentos. Revista Brasileira de Farmácia., v.87, n.3, p.89-91, 2006. 
ROCHA, Ana Leda Ribeiro da. Uso racional de medicamentos. 2014. 49 f. Trabalho de Conclusão de Curso (Especialização) - Instituto de Tecnologia em Fármacos/Farmanguinhos, Fundação Oswaldo Cruz, Rio de Janeiro, 2014.

WHO (WORLD HEALTH ORGANIZATION). How to investigate the use of medicines by consumers. 98p., 2004c.

WHO (WORLD HEALTH ORGANIZATION).Using indicators to measure country pharmaceutical situations Fact Book on WHO Level I and Level II monitoring indicators. 111p., $2006 a$.

Autor para correspondência:

Adriana Mary Mestriner Felipe de Melo

Email: mestriner@unigran.br

Centro Universitário da Grande Dourados (UNIGRAN)

Recebido: 27/02/2021 Aceite: 20/05/2021 